\title{
Which of MIS-TLIF and TLIF Can Leads to Better Pedicle Screws Placement Accuracy and Clinical Outcomes with Navigation Guidance?
}

JiaBin Liu

Third Military Medical University Second Affiliated Hospital: Xinqiao Hospital JunLong Wu

PLA: People's Liberation Army

Rui Zuo

Third Military Medical University Second Affiliated Hospital: Xinqiao Hospital

ChangQing Li

Third Military Medical University Second Affiliated Hospital: Xinqiao Hospital

Chao Zhang

Third Military Medical University Second Affiliated Hospital: Xinqiao Hospital

Yue Zhou ( $\nabla$ happyzhou@vip.163.com )

Xinqiao Hospital

Research article

Keywords: Computer-assisted navigation, minimally invasive surgery, pedicle screws implantation, spine surgery

Posted Date: May 28th, 2021

DOl: https://doi.org/10.21203/rs.3.rs-558088/v1

License: (c) (1) This work is licensed under a Creative Commons Attribution 4.0 International License. Read Full License 


\section{Abstract}

\section{Background}

Although previous studies have suggested that navigation can improve the accuracy of pedicle screw placement, there are still few studies comparing navigation-assisted transforaminal lumbar interbody fusion (TLIF) and navigation-assisted minimally-invasive TLIF (MIS-TLIF). The pedicle screw insertion entry point of navigation-assisted MIS-TLIF may be deflected from the planned entry point due to uneven bone-surface, which may result in misplacement. The purpose of this study was to explore the pedicle screws accuracy and clinical consequences of MIS-TLIF and TLIF both under 0-arm navigation to determine which surgical method is better.

\section{Methods}

A retrospective study of 54 patients who underwent single-segment navigation-assisted MIS-TLIF (NMTLIF) or navigation-assisted TLIF (N-TLIF) was conducted. In addition to the patient's demographic characteristics, intraoperative indicators and complications, the ODI and VAS scores were recorded and analyzed preoperatively, at 1, 6, 12 months and at the final follow-up postoperatively. The clinical accuracy and absolute accuracy of pedicle screw placement was assessed by postoperative CT. Multifidus muscle injury were evaluated by T2-weighted MRI.

\section{Results}

Compared with N-TLIF, NM-TLIF was more advantageous in the incision length, intraoperative blood loss, drainage volume, time before ambulation, length of hospital stays, blood transfusion rate and analgesia rate $(\mathrm{p}<0.05)$. The ODI and VAS for low back pain scores were better than those of N-TLIF at 1 month and 6 months after surgery $(p<0.05)$. There was no significant difference in the screw clinical qualitative accuracy $(97.3 \%$ vs. $96.2 \%, p>0.05)$. The absolute quantitative accuracy results show that the axial translational error, sagittal translational error and sagittal angle error of NM-TLIF group are significantly greater than that in N-TLIF group $(P<0.05)$. The mean $T 2$-weighted signal intensity of multifidus muscle in the NM-TLIF group was significantly lower than that in the N-TLIF group $(P<0.05)$

\section{Conclusions}

Compared with N-TLIF, NM-TLIF has more minimally invasive advantages, it does not yield a lower accuracy of screw placement and can achieve better symptom relief in the middle stage of postoperative recovery. However囚more attention on real-time adjustment should be paid to pedicle insertion in NM-TLIF, rather than just following the entry point and trajectory of the intraoperative plan.

\section{Background}

Transforaminal lumbar interbody fusion (TLIF) is an effective and widely accepted treatment for lumbar degenerative diseases ${ }^{1}$. However, conventional TLIFTLIF inevitably leads to a large incision, paravertebral 
muscle atrophy, massive blood loss, significant postoperative pain, and a long recovery time ${ }^{2}$.

Compared with TLIF, minimally invasive TLIF (MIS-TLIF) was demonstrated to have significant advantages, including a smaller incision, less severe paravertebral muscle dissection, less blood loss, less severe postoperative pain, and faster postoperative recovery ${ }^{3,4}$, and to yield comparable clinical results ${ }^{5 \text {, }}$ ${ }^{6}$. Nevertheless, due to the inadequate exposure of anatomical landmarks, traditional MIS-TLIF is often associated with insufficient decompression, the inaccurate placement of cages, and an increased risk of the malposition or perforation of pedicle screws ${ }^{7}$. Moreover, multiple fluoroscopies are required to ensure accurate pedicle screw placement, which is not only cumbersome but also increases the radiation exposure of patients and operating room staff members, which may increase the health risks ${ }^{8}$.

Following the introduction of cone-beam CT-guided spinal navigation, numerous reports in the literature have been published demonstrating its utility in increasing the accuracy of pedicle screw placement and decreasing the incidence of neurological injury from misplaced pedicle screws ${ }^{9,10}$. It also reduces the health risks associated with repeated fluoroscopy ${ }^{11}$.

With the use of navigation, the MIS-TLIF operation is more accurate, intelligent, and safe. However, in navigation-assisted MIS-TLIF (NM-TLIF), due to inadequate surrounding tissue dissection during the operation, the bone surface of the area in which the pedicle screw is placed is often uneven. The screw placement apparatus (e.g., the navigation drill guide, NDG) for NM-TLIF often cannot be fixed very well, which inevitably results in the puncture point and the trajectory being displaced. This issue may increase the risk of pedicle screw misplacement or perforation, resulting in surgical failure. In contrast, the surrounding tissues around the navigation-assisted TLIF (N-TLIF) area are fully exposed, and a definite screw setting point can be prepared by a rongeur to prevent the above issues to a certain extent (Fig. 1). Whether the effect of pedicle screw placement specifically in N-TLIF is better than that in NM-TLIF and yields better clinical efficacy has never been discussed. The purpose of this study was to compare the pedicle screw accuracy and clinical outcomes between NM-TLIF and N-TLIF under O-arm navigation guidance to determine which surgical method is better and guide clinical decision making.

\section{Methods}

\section{Patient Selection}

This study was approved by the ethics committee of the Third Military Medical University and conducted by a university-affiliated hospital in a major Chinese city. The medical records of patients who underwent single-level posterior lumbar interbody fusion guided by 0-arm-based navigation between May 2015 and May 2019 were selected. The study is based on a retrospective analysis of prospectively collected data. The patients were divided into the N-TLIF group and NM-TLIF group according to whether minimally invasive procedures or open procedures were implemented during the operation. The inclusion criteria of this study were patients with mainly complaints of low back pain; varying degrees of radicular pain and neurologic symptoms; single-level lumbar disc herniation or stenosis with one-grade lumbar 
spondylolisthesis, as demonstrated by anteroposterior, lateral, oblique, and flexion-extension plain radiographs, computed tomography (CT) scans, and magnetic resonance imaging (MRI) scans; and a lack of response to extensive conservative therapy for at least 3 months before surgery. The exclusion criteria included patients with trauma, active infections, tumors, spinal deformities, multisegmental fusions, and a history of fusion. The clinical data of all patients were obtained from medical records and telephone follow-ups.

\section{Clinical Evaluation}

Patient age, gender, body mass index (BMI), American Society of Anesthesiologists (ASA) class, preoperative diagnoses, operation level, incision length, operating time, intraoperative blood loss, drainage volume, time before ambulation, analgesic use rate, blood transfusion rate, hospitalization stay, hospitalization cost and complications were assessed and recorded. Follow-ups, including clinical evaluations, were performed preoperatively and 1 month, 6 months, and 12 months after surgery. The minimum follow-up time was 12 months. The visual analog scale (VAS) score was used to evaluate pain in the lower back and lower extremities. The Oswestry disability index (ODI) was used to evaluate patients' daily life functions.

\section{Radiological Evaluations}

Clinical qualitative accuracy was performed after the drainage tube was removed, including anteriorposterior and transverse flexural and telescopic ordinary X-ray, CT. The positions of the pedicle screws were evaluated by postoperative CT scans in all cases. The screw position was graded from 0 to 3 , and screws that did not breach received a Grade 0. For the screws that did breach, Grade 1 was assigned to minor breaches of less than $2 \mathrm{~mm}$, Grade 2 was assigned to breaches between 2 and $4 \mathrm{~mm}$, and Grade 3 was assigned to breaches more than $4 \mathrm{~mm}^{12}$.

The absolute quantitative accuracy of each pedicle screw was measured using the method reported by Guha D., et al. ${ }^{13}$. The final screw position on postoperative CT imaging was compared with the screenshot of the ideal screw entry point and trajectory planned by the intraoperative navigation system. Translational and angular deviations from the planned entry point and trajectory was measured in the axial and sagittal planes. In the axial plane, positive translational deviations denote a lateral deflection of the entry point, and positive angular deviations denote a more lateral trajectory. In the sagittal plane, positive translational deviations denote a superior deflection of the entry point, and positive angular deviations denote a more cranial trajectory. All image processing and measurement are performed using the Mimics software (Version 21, Materialise, Belgium).

An MRI scan taken one year after surgery will be used to evaluate the multifidus muscle injury at the surgical site ${ }^{14}$. The multifidus muscle injury was assessed using a Picture Archiving and Communication System (PACS) workstation. In the axial images, MRI T2-weighted grayscale values of the psoas major and multifidus muscles were measured in a $300 \mathrm{~mm} 2$ circular region of interest. MRI T2-weighted grayscale values at the surgical segment and adjacent segments were measured, and their mean values 
represented the mean signal intensity of the psoas major and multifidus muscle, respectively. The T2weighted signal intensity ratio was determined by dividing the mean signal intensity of the psoas major by the mean signal intensity of the multifidus muscle. The assessment of the images and measurements of the screw position were performed by a surgeon who was not involved in the study.

\section{Surgical Procedure}

\section{NM-TLIF Procedure}

After general anesthesia, the patient was positioned prone on the operating table. A 3.5-4 cm longitudinal incision was made $3 \mathrm{~cm}$ lateral to the midline on the decompression side for the placement of the extensible retractor (Mast Quadrant Retractor System). The facet joints corresponding to the target intervertebral space were exposed. The entire facet joint and ligamentum flavum were removed to expose the nerve roots and dural sac. The adhered nerve roots were separated, and the nerve root canal was decompressed. Then, complete discectomy was performed. The autogenous bone obtained during decompression was placed in the prepared intervertebral disc space, and the cage filled with bone fragments was placed obliquely. Two Kirschner wires were implanted into the posterior superior iliac spine of the patient, and the reference frame was fixed on it. The CT images of the surgical area were obtained by the 0-arm and then transferred to the navigation system host (StealthStation S7 surgical navigation system, Medtronic) by a network cable for intraoperative three-dimensional reconstruction. After the reconstructed image was obtained, the instrument was registered, and the accuracy of navigation was initially assessed by placing the probe on the spinous process and observing whether the image corresponded to the navigation image. Then, the entry point and trajectory of the pedicle screw were planned in the sagittal and axial views of the navigation system, and save a screenshot of the plan. Then place the guide wire with the electric drill under the guidance of the navigation drill guide (Fig. 2). After the implantation of all four guide wires, fluoroscopy was used to verify the consistency between the guide wires and the intraoperative plan. After 4 pedicle screws were inserted along the verified guide wire, the accuracy of the screws was finally verified by fluoroscopy. Then, titanium rods were inserted to connect the pedicle screws. The intervertebral space was pressurized, and the nut was tightened.

\section{N-TLIF Procedure}

After general anesthesia, the patient was placed in the prone position, and a median incision of approximately $8 \mathrm{~cm}$ was made with the target intervertebral space as the center. The paravertebral muscles were stripped, and the spinous process of the upper vertebral body was exposed. The spinous process reference frame was fixed on the spinous process. 0-arm scanning was performed to reconstruct the image of the operation area. Pedicle screws were implanted under the assistance of 0-arm navigation. After the entry point and trajectory were planned and the screenshot is saved, some bone cortex was removed with a rongeur at the planned position to make space for the definite insertion point. The subsequent implantation process was consistent with that performed for NM-TLIF, and the position was confirmed by fluoroscopy. Intervertebral space distraction was performed after bilateral titanium rod implantation. Target space laminectomy decompression, nerve root release, intervertebral space 
treatment and bone graft fusion were performed. Fluoroscopy confirmed that the location of the cage was ideal. The position of the connecting rod was properly adjusted, and the nut was properly pressurized and locked.

\section{Statistical Analyses}

Statistical analyses were performed with SPSS (version 23.0, SPSS Inc., Chicago, IL, USA). Two sets of continuous variables were compared by the student's $t$ test or the two-sample Mann-Whitney $U$ test. The categorical variables were compared between the two groups by the chi-square test or Fisher's exact test. The ODI and VAS scores were analyzed by repeated measures ANOVA. $\mathrm{P}<0.05$ was considered statistically significant.

\section{Results}

\section{Patients Characteristics}

The data from 28 patients who underwent NM-TLIF and 26 patients who underwent N-TLIF were included in this analysis, and all cases were treated with single-segment pedicle screw fixation guided by 0 -armbased navigation. The demographic characteristics were comparable between the two groups. There were no significant differences in age, gender, BMI, ASA classification or preoperative diagnosis between the two groups. All patients underwent surgery for degenerative diseases (spondylolisthesis, lumbar instability, lumbar spinal stenosis or lumber disc herniation). All patients were followed up for at least 12 months (Table 1).

\section{Clinical Evaluations}

There were no statistically significant differences between the two groups in the proportions of surgery levels, operative time, hospitalization stay or operative cost. The incision length in the NM-TLIF group was significantly shorter than that in the N-TLIF group $(P<0.05)$, intraoperative blood loss and postoperative drainage volume were significantly lower in the NM-TLIF group than in the N-TLIF group $(P<0.05)$, and the drainage tube was pulled out approximately one day later in the N-TLIF group than in the NM-TLIF group $(\mathrm{P}<0.05)$. Four patients in the N-TLIF group required blood transfusion $(15.4 \%)$, while no patients in the NM-TLIF group needed blood transfusion. The postoperative analgesia rate was also significantly higher in the N-TLIF group $(P<0.05)$ (Table 2$)$.

There were no significant differences in the ODI score, low back pain VAS score or lower limb pain VAS score between the two groups before surgery $(P>0.05)$. These indicators showed a gradually decreasing trend in the two groups postoperatively. The ODI score and low back pain VAS score were significantly lower in the NM-TLIF group at 1 and 6 months postoperatively $(P<0.05)$. There were no significant differences in the VAS score for postoperative lower extremity pain $(P>0.05)$. Additionally, there was no significant difference between the two groups in the three indicators at the final follow-up $(P>0.05)$ (Table 3). In all cases, instrument-related complications (such as nerve root injury, cerebrospinal fluid leakage, 
and visceral injury) were not observed, and revision surgery was not required due to pedicle screw malposition.

\section{Radiological Evaluations}

In this study, the 216 pedicle screws were graded based on the postoperative CT images, 112 of the screws were implanted in the NM-TLIF group, and 104 were implanted in the N-TLIF group. The clinical qualitative accuracy rates (Grades $0 \& 1$ ) of the two groups were $97.3 \%$ (NM-TLIF) and $96.2 \%$ (N-TLIF). There were 3 screws in the NM-TLIF group and 4 screws in the N-TLIF group that were not aligned (Grade 2), but none of the patients showed symptoms of spinal cord or nerve injury. None of the pedicle screws in Grade 3 were observed. There was no significant difference in the accuracy rate between the two groups $(p>0.05)$ (Table 3$)$.

The absolute quantitative accuracy results show that the axial translational error, sagittal translational error and sagittal angle error of NM-TLIF group are significantly greater than that in N-TLIF group ( $P$ $<0.05)$. Compared with the N-TLIF group, the entry point of the NM-TLIF group is more prone to lateral deflection and inferior deflection. Meanwhile, NM-TLIF is prone to obtain more cranial trajectory. But axial Angle error are not statistically significant $(P>0.05)$ (Table 5).

One year after surgery, muscle atrophy and fat infiltration were observed in both groups (Fig. 3). The mean T2-weighted signal intensity of multifidus muscle in the NM-TLIF group was significantly lower than that in the N-TLIF group $(P<0.05)$ (Fig. 4).

\section{Discussion}

This retrospective cohort study reveals that N-TLIF does not have better accuracy or clinical outcomes than does NM-TLIF. In this study, the clinical results of 54 patients and the accuracy of 206 pedicle screws were retrospectively analyzed, and it was found that the two different surgical methods could yield excellent screw placement accuracy with the assistance of navigation, and there was no significant difference between them. Both of them can effectively alleviate the symptoms of patients and yield similar clinical outcomes. It is worth noting that the advantages of minimally invasive surgery in NM-TLIF cannot be ignored.

Lumbar degenerative disease is a common disease among elderly individuals. For patients who fail to respond to regular conservative treatment, TLIF is an effective treatment method. It can achieve bilateral intervertebral fusion by entering the spinal canal from one side, and the central canal is not affected in the operation, reducing the occurrence of cerebrospinal fluid leakage. There is no need to pull the nerve root or dural sac to reduce the probability of nerve injury. The contralateral vertebral plate and facet joints are retained, and the upper edge of the vertebral plate, spinous process, superior spinous ligament and interspinous ligament are retained, and these structures have little influence on the stability of the spine

${ }^{15}$. MIS-TLIF is a minimally invasive version of TLIF. Compared with TLIF, MIS-TLIF yields the same 
surgical effect and has the advantages of a smaller incision, less blood loss, less severe soft tissue injury, a quick recovery and a short hospital stay ${ }^{16}$.

Computer-assisted navigation can provide a holistic anatomical image of the surgical area through a single scan, prevent repeated fluoroscopy during the operation, and locate and track the relative position of surgical instruments and anatomical structures in real time, thus allowing more accurate fusion cage and pedicle screw placement ${ }^{17}$. Intraoperative navigation can reduce the surgical difficulty caused by the small visual field and the difficulty in recognizing anatomical structures during MIS-TLIF surgery, reduce the radiation exposure and health risks caused by repeated fluoroscopic positioning during traditional minimally invasive surgery, and significantly improve the learning curve ${ }^{18,19}$. As with TLIF, intraoperative navigation significantly improves the accuracy of MIS-TLIF pedicle screw placement and reduces intraoperative complications ${ }^{10,20,21}$. Compared with freehand instrumentation, intraoperative navigation can also significantly improve the one-time accuracy of the insertion of pedicle screws and can be used to prevent injury to the pedicle and surrounding structures from screw reinsertion ${ }^{22,23}$. The purpose of this study was to discuss the effects of two different navigation-assisted surgical procedures, TLIF and MIS-TLIF, especially regarding the accuracy of pedicle screw implantation and clinical outcomes.

With 0-arm navigation, the intraoperative screw trajectory image can be seen in real time, thus making the implantation process more accurate and safer in the NM-TLIF group ${ }^{24,25}$. In our study, two methods of surgery involving intraoperative 0-arm navigation assistance yielded high accuracy, with no statistically significant difference between the methods ( $97.3 \%$ vs. $96.2 \%, p>0.05$ ). The results showed that the accuracy of NM-TLIF was not inferior to that of N-TLIF due to insufficient anatomical standard exposure or other reasons, which was consistent with previous research results ${ }^{18,26}$. Significantly, the absolute accuracy results showed that both axial and sagittal translational errors were greater in the NM-TLIF group than in the N-TLIF group, which verifies our hypothesis at the beginning of the article. However, despite the larger absolute accuracy error at the entry point in the NM-TLIF group, there was no statistically significant difference in the final accuracy of pedicle screw placement between the two groups. This result suggests that there is no direct correlation between pedicle screw placement accuracy and absolute accuracy of the entry point deflection, which is consistent with the results of previous study ${ }^{13}$. We believe that this finding is because the visualization of navigation can not only be used for planning when using the NDG to find the entry point, but also be used to adjust the trajectory direction when an electric drill is used to obtain the trajectory. Even when the entry point slips slightly because the NDG is not firmly fixed on the bone surface, the surgeon can adjust the trajectory through the screen after the drill bit enters the bone cortex to achieve good placement of the pedicle screws. In view of the fact that the NM-TLIF screw entry point is more likely to be deflected, we suggest that more attention should be paid to the entire NM-TLIF screw setting process, rather than just following the entry point and trajectory of the intraoperative plan, and the screw orientation should be adjusted at any time during screw setting. 
According to our results, the advantages of minimally invasive surgery were revealed in this study. In the NM group, the incision length, intraoperative blood loss, postoperative drainage volume, time before ambulation, blood transfusion rate, postoperative analgesia rate and hospital stay time were significantly reduced. For the clinical outcomes, within at least 12 months of follow-up, the postoperative lumbar pain VAS, lower extremity pain VAS and ODI scores improved significantly in the two groups of patients. There were no significant differences in the intraoperative or postoperative complications between the two groups. Both open and minimally invasive procedures, with the assistance of navigation, can yield consistently good results. However, the use of intraoperative navigation increased the duration of the operation compared with that reported in our previous studies at the same institution ${ }^{27}$, which is consistent with the results of previous research ${ }^{28}$. According to findings reported by other authors, extra time required for the operation is mainly required for the preparation of the navigation equipment, whereas the screw implant time is dramatically reduced, so the operation process is improved with navigation 29,30 .

In this study, N-TLIF did not yield better screw placement accuracy or better clinical outcomes; in contrast, NM-TLIF seemed to have more advantages. NM-TLIF not only has the above advantages of minimally invasive surgery but also causes significantly less damage to the surrounding spinal muscle tissues than does N-TLIF, and NM-TLIF is superior to N-TLIF regarding long-term muscle tissue recovery. Serban, D. et al. ${ }^{31}$ divided patients into a standard TLIF group and MIS-TLIF group. The results showed that the magnitude of improvement in the ODI score from before to after surgery was statistically significant in both groups and similar between groups. The two techniques provided similar clinical and radiological outcomes at 1 year. The patients undergoing MIS-TLIF had a shorter hospital stay. Their results are similar to ours. This finding also demonstrates the advantages of MIS-TLIF.

Radiation exposure during surgery has always been the focus of spinal surgeons. The use of intraoperative navigation can reduce the radiation exposure of patients and surgeons, as has been reported in previous articles. Araiza E.T. et al found that screw placement with the C-arm exposed the surgeon to a significantly greater amount of radiation $(3.87 \times 10$ rads vs. $0.32 \times 10, P<0.001)$, while the 0 -arm exposed the cadaver to a significantly greater amount of radiation ( 0.03 vs. 2.76 rads, $P<0.001)$ 11. In our study, the levels of radiation exposure to the patients, surgeons, and operating room staff members were not specifically discussed because all patients underwent a complete 0-arm CT scan. All patients strictly followed the same procedure for pedicle screw implantation, and no patients underwent a second CT scan due to navigation accuracy problems, so we assumed that the radiation dose was approximately the same between the two groups. The surgeon and the operating room staff members leave the operating room during CT or X-ray scans, so in our opinion, the level of radiation they are exposed to is negligible, but the patient might be exposed to more radiation ${ }^{32}, 33$.

The main limitations of this study are as follows: this study was a retrospective study, with a low level of evidence and the possibility of selection bias. No previous studies have compared the levels of radiation exposure specifically across different methods, and only a general assessment of the differences in 
relative radiation between the prescribed procedures was reported, but the absolute value could not be obtained or compared with those of other methods. Prospective randomized controlled studies with larger sample sizes should be carried out in the future to overcome the limitations of existing studies.

Advancements in both minimally invasive surgery and navigation technology will lead to improvements in lumbar fusion surgery in the future. In the future, navigation technologies should have improved accuracy and lower levels of image drift, and severe complications caused by imaging errors should not occur. Furthermore, the operation process should be optimized, the efficiency of navigation surgery should be improved, the operation time should be shortened, the cost to use the navigation system should be reduced, and the method should be used more widely. In addition, it is also necessary to carry out multicenter, large-sample prospective research studies to obtain a high level of evidence.

\section{Conclusion}

When the MIS-TLIF is navigation-assisted, the surgeon can compensate in real time for the trajectory displacement of the pedicle screw due to the uneven of the insertion point, it does not reduce the accuracy of pedicle screw implantation. Compared with N-TLIF group, NM-TLIF group has achieved comparable clinical results, with additional minimally invasive benefits of shorter incision length, less intraoperative blood loss, smaller postoperative drainage volume, earlier ambulation time, lower blood transfusion rate, less postoperative analgesia rate, and shorter hospital stay. Not only that, NM-TLIF can even achieve better symptom relief in the middle course of the disease. However,more attention on realtime adjustment should be paid to pedicle insertion in NM-TLIF surgery, rather than just following the entry point and trajectory of the intraoperative plan.

\section{Abbreviations}

ASA: American Society of Anesthesiologists

BMl: Body mass index

CT: Computed tomography

MIS-TLIF: Minimally invasive transforaminal lumbar interbody fusion

MRI: Magnetic resonance imaging

NM-TLIF: Navigation-assisted minimally invasive transforaminal lumbar interbody fusion

N-TLIF: Navigation-assisted transforaminal lumbar interbody fusion

NDG: Navigation drill guide

ODI: Oswestry disability index 
TLIF: Transforaminal lumbar interbody

VAS: Visual analog scale

3D: Three dimensional

\section{Declarations}

\section{Ethics approval and consent to participate}

This study was approved by the Medical Ethics Committee of Xinqiao hospital, Army Medical University, which was performed in accordance with the ethical standards of the 1964 Declaration of Helsinki and its later amendments or comparable ethical standards. The patients received a thorough explanation of this study, and their oral and written informed consent was obtained in this study.

\section{Consent for publication}

Not applicable.

\section{Availability of data and materials}

The datasets used and analysed during the current study are available from the corresponding author on reasonable request.

\section{Competing interests}

The authors declare that they have no competing interests.

\section{Funding}

Not applicable

\section{Authors' contributions}

JL collected, analyzed and interpreted the patients' clinical data, and was a major contributor in writing the manuscript. JW provided methodological guidance and validation of the data, as well as proofreading and revision of the manuscript. RZ measured and analyzed the image data. CL provides data resources of patients and guided the research. $\mathrm{CZ}$ carried out this project and guided the research. $\mathrm{YZ}$ conceived and designed the research. All authors read and approved the final manuscript.

\section{Acknowledgements}

Not applicable.

\section{References}


1. de Kunder S.L., van Kuijk S.M.J., Rijkers K., et al. Transforaminal lumbar interbody fusion (TLIF) versus posterior lumbar interbody fusion (PLIF) in lumbar spondylolisthesis: a systematic review and meta-analysis. Spine J. 2017;17:1712-1721, https://doi.org/10.1016/j.spinee.2017.06.018.

2. Ge D.H., Stekas N.D., Varlotta C.G., et al. Comparative Analysis of Two Transforaminal Lumbar Interbody Fusion Techniques: Open TLIF Versus Wiltse MIS TLIF. Spine (Phila Pa 1976). 2019;44:E555-E560, https://doi.org/10.1097/BRS.0000000000002903.

3. Chen K., Chen H., Zhang K., et al. O-arm Navigation Combined With Microscope-assisted MIS-TLIF in the Treatment of Lumbar Degenerative Disease. Clin Spine Surg. 2019;32:E235-E240, https://doi.org/10.1097/BSD.0000000000000804.

4. Qin R., Liu B., Zhou P., et al. Minimally Invasive Versus Traditional Open Transforaminal Lumbar Interbody Fusion for the Treatment of Single-Level Spondylolisthesis Grades 1 and 2: A Systematic Review and Meta-Analysis. World Neurosurg. 2019;122:180-189, https://doi.org/10.1016/j.wneu.2018.10.202.

5. Mummaneni P.V., Bisson E.F., Kerezoudis P., et al. Minimally invasive versus open fusion for Grade I degenerative lumbar spondylolisthesis: analysis of the Quality Outcomes Database. Neurosurg Focus. 2017;43:E11, https://doi.org/10.3171/2017.5.FOCUS17188.

6. Jin-Tao Q., Yu T., Mei W., et al. Comparison of MIS vs. open PLIF/TLIF with regard to clinical improvement, fusion rate, and incidence of major complication: a meta-analysis. Eur Spine J. 2015;24:1058-65, https://doi.org/10.1007/s00586-015-3890-5.

7. Tian W., Liu Y., Fan M., et al. CAMISS Concept and Its Clinical Application. Adv Exp Med Biol. 2018;1093:31-46, https://doi.org/10.1007/978-981-13-1396-7_3.

8. Navarro-Ramirez R., Lang G., Lian X., et al. Total Navigation in Spine Surgery; A Concise Guide to Eliminate Fluoroscopy Using a Portable Intraoperative Computed Tomography 3-Dimensional Navigation System. World Neurosurg. 2017;100:325-335, https://doi.org/10.1016/j.wneu.2017.01.025.

9. Fomekong E., Pierrard J. and Raftopoulos C. Comparative Cohort Study of Percutaneous Pedicle Screw Implantation without Versus with Navigation in Patients Undergoing Surgery for Degenerative Lumbar Disc Disease. World Neurosurg. 2018;111:e410-e417, https://doi.org/10.1016/j.wneu.2017.12.080.

10. Silbermann J., Riese F., Allam Y., et al. Computer tomography assessment of pedicle screw placement in lumbar and sacral spine: comparison between free-hand and 0-arm based navigation techniques. Eur Spine J. 2011;20:875-81, https://doi.org/10.1007/s00586-010-1683-4.

11. Araiza E.T., Medda S., Plate J.F., et al. Comparing the Efficiency, Radiation Exposure, and Accuracy Using C-Arm versus 0-Arm With 3D Navigation in Placement of Transiliac-Transsacral and lliosacral Screws: A Cadaveric Study Evaluating an Early Career Surgeon. J Orthop Trauma. 2020;34:302-306, https://doi.org/10.1097/bot.0000000000001724.

12. Rampersaud Y.R., Pik J.H., Salonen D., et al. Clinical accuracy of fluoroscopic computer-assisted pedicle screw fixation: a CT analysis. Spine (Phila Pa 1976). 2005;30:E183-90, 
https://doi.org/10.1097/01.brs.0000157490.65706.38.

13. Guha D., Jakubovic R., Gupta S., et al. Spinal intraoperative three-dimensional navigation: correlation between clinical and absolute engineering accuracy. Spine J. 2017;17:489-498, https://doi.org/10.1016/j.spinee.2016.10.020.

14. Lehmann Urban D., Mohamed M., Ludolph A.C., et al. The value of qualitative muscle MRI in the diagnostic procedures of myopathies: a biopsy-controlled study in 191 patients. Ther Adv Neurol Disord. 2021;14:1756286420985256, https://doi.org/10.1177/1756286420985256.

15. Reid P.C., Morr S. and Kaiser M.G. State of the union: a review of lumbar fusion indications and techniques for degenerative spine disease. J Neurosurg Spine. 2019;31:1-14, https://doi.org/10.3171/2019.4.Spine18915.

16. Fessler R.G., Smith Z.A., Slimack N., et al. Current advances and evidence in minimally invasive spine surgery. Minim Invasive Surg. 2012;2012:508415, https://doi.org/10.1155/2012/508415.

17. Patel V.V. Opportune Situations for Spine Surgical Navigation. Spine (Phila Pa 1976). 2017;42 Suppl 7:S27, https://doi.org/10.1097/brs.0000000000002037.

18. Xu Y.F., Le X.F., Tian W., et al. Computer-assisted, minimally invasive transforaminal lumbar interbody fusion: One surgeon's learning curve A STROBE-compliant article. Medicine (Baltimore). 2018;97:e11423, https://doi.org/10.1097/MD.0000000000011423.

19. Fomekong E., Safi S.E. and Raftopoulos C. Spine Navigation Based on 3-Dimensional Robotic Fluoroscopy for Accurate Percutaneous Pedicle Screw Placement: A Prospective Study of 66 Consecutive Cases. World Neurosurg. 2017;108:76-83, https://doi.org/10.1016/j.wneu.2017.08.149.

20. Wu M.H., Dubey N.K., Li Y.Y., et al. Comparison of minimally invasive spine surgery using intraoperative computed tomography integrated navigation, fluoroscopy, and conventional open surgery for lumbar spondylolisthesis: a prospective registry-based cohort study. Spine J. 2017;17:1082-1090, https://doi.org/10.1016/j.spinee.2017.04.002.

21. Tian W., Xu Y.F., Liu B., et al. Computer-assisted Minimally Invasive Transforaminal Lumbar Interbody Fusion May Be Better Than Open Surgery for Treating Degenerative Lumbar Disease. Clin Spine Surg. 2017;30:237-242, https://doi.org/10.1097/bsd.0000000000000165.

22. Tajsic T., Patel K., Farmer R., et al. Spinal navigation for minimally invasive thoracic and lumbosacral spine fixation: implications for radiation exposure, operative time, and accuracy of pedicle screw placement. Eur Spine J. 2018;27:1918-1924, https://doi.org/10.1007/s00586-018-5587-z.

23. Xu Y.F., Zhang Q., Le X.F., et al. Comparison of the One-Time Accuracy of Simulated Freehand and Navigation Simulated Pedicle Screw Insertion. World Neurosurg. 2019;128:e347-e354, https://doi.org/10.1016/j.wneu.2019.04.151.

24. Ling J.M., Dinesh S.K., Pang B.C., et al. Routine spinal navigation for thoraco-lumbar pedicle screw insertion using the 0 -arm three-dimensional imaging system improves placement accuracy. J Clin Neurosci. 2014;21:493-8, https://doi.org/10.1016/j.jocn.2013.02.034.

25. Houten J.K., Nasser R. and Baxi N. Clinical assessment of percutaneous lumbar pedicle screw placement using theO-arm multidimensional surgical imaging system. Neurosurgery. 2012;70:990-5, 
https://doi.org/10.1227/NEU.0b013e318237a829.

26. Yadav R.I., Long L. and Yanming C. Comparison of the effectiveness and outcome of microendoscopic and open discectomy in patients suffering from lumbar disc herniation. Medicine (Baltimore). 2019;98:e16627, https://doi.org/10.1097/MD.0000000000016627.

27. Wu J., Zhang C., Lu K., et al. A Novel Inextensible Endoscopic Tube Versus Traditional Extensible Retractor System in Single-Level Minimally Invasive Transforaminal Lumbar Interbody Fusion: A Prospective Observation Study. Pain Physician. 2019;22:E587-E599,

28. Wagner S.C., Morrissey P.B., Kaye I.D., et al. Intraoperative pedicle screw navigation does not significantly affect complication rates after spine surgery. J Clin Neurosci. 2018;47:198-201, https://doi.org/10.1016/j.jocn.2017.09.024.

29. Balling H. Learning curve analysis of 3D-fluoroscopy image-guided pedicle screw insertions in lumbar single-level fusion procedures. Arch Orthop Trauma Surg. 2018;138:1501-1509, https://doi.org/10.1007/s00402-018-2994-x.

30. Balling H. Time Demand and Radiation Dose in 3D-Fluoroscopy-based Navigation-assisted 3DFluoroscopy-controlled Pedicle Screw Instrumentations. Spine (Phila Pa 1976). 2018;43:E512-e519, https://doi.org/10.1097/brs.0000000000002422.

31. Serban D., Calina N. and Tender G. Standard versus Minimally Invasive Transforaminal Lumbar Interbody Fusion: A Prospective Randomized Study. Biomed Res Int. 2017;2017:7236970, https://doi.org/10.1155/2017/7236970.

32. Bratschitsch G., Leitner L., Stücklschweiger G., et al. Radiation Exposure of Patient and Operating Room Personnel by Fluoroscopy and Navigation during Spinal Surgery. Sci Rep. 2019;9:17652, https://doi.org/10.1038/s41598-019-53472-z.

33. Pitteloud N., Gamulin A., Barea C., et al. Radiation exposure using the 0-arm(®) surgical imaging system. Eur Spine J. 2017;26:651-657, https://doi.org/10.1007/s00586-016-4773-0.

\section{Tables}


Table 1

Patients Characteristics

\begin{tabular}{|llll|}
\hline & $\begin{array}{l}\text { NM-TLIF } \\
(\mathrm{n}=28)\end{array}$ & $\begin{array}{l}\text { N-TLIF } \\
(\mathrm{n}=26)\end{array}$ & $\mathrm{p}$-Value \\
\hline Demographics & & & \\
\hline Age (years) & $52.1 \pm 12.1$ & $54.5 \pm 13.7$ & 0.525 \\
\hline Female gender* & $16(57.1 \%)$ & $15(57.7 \%)$ & 0.967 \\
\hline Body mass index (kg/m², BMI) & $23.0 \pm 2.6$ & $24.0 \pm 2.7$ & 0.663 \\
\hline ASA class $\ddagger$ & $1.2 \pm 0.42$ & $1.3 \pm 0.47$ & 0.438 \\
\hline Diagnosis & & & \\
\hline Spondylolisthesis* & $12(42.9 \%)$ & $14(53.8 \%)$ & 0.419 \\
\hline Lumbar Instability & $10(35.7 \%)$ & $6(23.1 \%)$ & 0.310 \\
\hline Lumbar Spinal Stenosis $\dagger$ & $4(14.3 \%)$ & $5(19.2 \%)$ & 0.626 \\
\hline Lumbar Disc Herniation* & $2(7.1 \%)$ & $1(3.8 \%)$ & 0.597 \\
\hline Follow up(month) & & & \\
\hline Range & $13-59$ & $12-59$ & \\
\hline Mean & $39.5 \pm 6.1$ & $37.0 \pm 6.7$ & 0.544 \\
\hline
\end{tabular}

${ }^{*}$ Chi-squared test. †Fisher's exact test. ‡Two-Sample Mann-Whitney U Test. Otherwise, independentsamples $t$ test with equal variances assumed 
Table 2

Surgical data

\begin{tabular}{|c|c|c|c|}
\hline & $\begin{array}{l}\text { MN-TLIF } \\
(n=28)\end{array}$ & $\begin{array}{l}\text { N-TLIF } \\
(n=26)\end{array}$ & $\mathrm{p}$-Value \\
\hline \multicolumn{4}{|l|}{ Parameter Overall } \\
\hline \multicolumn{4}{|l|}{ Operation level, $n(\%)$} \\
\hline L3-L4† & $1(3.6 \%)$ & $2(7.7 \%)$ & 0.604 \\
\hline L4-L5* & $18(64.3 \%)$ & $17(65.4 \%)$ & 0.933 \\
\hline L5-S1* & $9(32.1 \%)$ & $7(26.9 \%)$ & 0.675 \\
\hline \multicolumn{4}{|l|}{ Perioperative indicators } \\
\hline Incision lengths $(\mathrm{cm}) \ddagger$ & $4.1 \pm 1.2$ & $7.8 \pm 1.0$ & $<0.001$ \\
\hline Intraoperative blood loss $(\mathrm{ml}) \ddagger$ & $191.4 \pm 281.7$ & $328.8 \pm 303.4$ & 0.017 \\
\hline Operative time $(\mathrm{min})$ & $193.8 \pm 57.9$ & $195.0 \pm 56.9$ & 0.558 \\
\hline Drainage volume $(\mathrm{ml}) \ddagger$ & $64.6 \pm 65.8$ & $186.8 \pm 150.0$ & $<0.001$ \\
\hline Time to ambulation (day) $\ddagger$ & $2.1 \pm 0.3$ & $3.2 \pm 1.3$ & $<0.001$ \\
\hline Hospitalization stay (day)‡ & $6.1 \pm 2.8$ & $8.5 \pm 3.3$ & 0.002 \\
\hline Blood transfusion rate§ & $0(0 \%)$ & $4(15.4 \%)$ & \\
\hline Analgesia ratio* & $3(10.7 \%)$ & $12(46.2 \%)$ & 0.004 \\
\hline Hospitalization cost (CNY)‡ & $61677.6 \pm 32991.7$ & $72397.1 \pm 20184.2$ & 0.665 \\
\hline
\end{tabular}

${ }^{*}$ Chi-squared test. †Fisher's exact test. łTwo-Sample Mann-Whitney U Test.乌lln 4 cases, intraoperative blood loss was more than $800 \mathrm{ml}$, up to $1200 \mathrm{ml}$, and hemoglobin concentration in all cases was lower than $70 \mathrm{~g} / \mathrm{L}$, which met the conditions for blood transfusion. 
Table 3

Comparison of clinical parameters between two groups.

\begin{tabular}{|llll|}
\hline & $\begin{array}{l}\text { NM-TLIF } \\
(\mathrm{n}=28)\end{array}$ & $\begin{array}{l}\text { N-TLIF } \\
(\mathrm{n}=26)\end{array}$ & $\mathrm{p}$-Value \\
\hline ODI scores & & & 0.121 \\
\hline Preoprative & $49.4 \pm 4.7$ & $50.3 \pm 5.9$ & 0.548 \\
\hline Postoperative 1 month & $20.5 \pm 6.7$ & $25.7 \pm 8.2$ & 0.013 \\
\hline Postoperative 6 month & $16.1 \pm 5.9$ & $19.8 \pm 6.9$ & 0.040 \\
\hline Postoperative 12 month & $12.8 \pm 5.7$ & $14.0 \pm 6.1$ & 0.478 \\
\hline Final follow-up & $8.0 \pm 5.2$ & $9.8 \pm 5.4$ & 0.345 \\
\hline Low back pain VAS scores & & & 0.006 \\
\hline Preoprative & $6.9 \pm 1.0$ & $7.3 \pm 1.3$ & 0.200 \\
\hline Postoperative 1 month & $2.9 \pm 1.0$ & $3.6 \pm 0.8$ & 0.006 \\
\hline Postoperative 6 month & $1.6 \pm 0.8$ & $2.3 \pm 0.9$ & 0.006 \\
\hline Postoperative 12 month & $1.2 \pm 0.9$ & $1.6 \pm 0.9$ & 0.094 \\
\hline Final follow-up & $0.7 \pm 0.7$ & $0.9 \pm 0.8$ & 0.384 \\
\hline Lower extremity pain VAS scores & & & 0.865 \\
\hline Preoprative & $5.3 \pm 1.7$ & $5.9 \pm 1.6$ & 0.211 \\
\hline Postoperative 1 month & $1.5 \pm 0.9$ & $1.4 \pm 0.7$ & 0.733 \\
\hline Postoperative 6 month & $1.4 \pm 1.0$ & $1.2 \pm 1.2$ & 0.361 \\
\hline Postoperative 12 month & $1.1 \pm 0.9$ & $1.0 \pm 1.0$ & 0.672 \\
\hline Final follow-up & $0.3 \pm 0.5$ & $0.4 \pm 0.6$ & 0.660 \\
\hline
\end{tabular}

ANOVA for repeated design data 
Table 4

Clinical quality accuracy of pedicle screws

\begin{tabular}{|c|c|c|c|c|}
\hline Level treated & Grade 0 & Grade 1 & Grade 2 Grade 3 & $\begin{array}{l}\text { Accuracy rate } \\
\text { (Grade } 0 \& 1 \text { ) }\end{array}$ \\
\hline \multicolumn{5}{|l|}{$\begin{array}{l}\text { NM-TLIF } \\
(n=112)\end{array}$} \\
\hline L3 & 2 & & & $2(100 \%)$ \\
\hline L4 & 35 & 2 & 1 & $37(97.4 \%)$ \\
\hline L5 & 48 & 4 & 2 & $52(96.3 \%)$ \\
\hline S1 & 15 & 3 & & $18(100 \%)$ \\
\hline Total & 100 & 9 & 3 & $109(97.3 \%)^{\star}$ \\
\hline \multicolumn{5}{|l|}{$\begin{array}{l}\text { N-TLIF } \\
(n=104)\end{array}$} \\
\hline L3 & 3 & 1 & & $4(100 \%)$ \\
\hline L4 & 36 & 1 & 1 & $37(97.4 \%)$ \\
\hline L5 & 43 & 3 & 2 & $46(95.8 \%)$ \\
\hline S1 & 12 & 1 & 1 & $13(92.9 \%)$ \\
\hline Total & 94 & 6 & 4 & $100(96.2 \%)^{\star}$ \\
\hline$p$-Value* & & & & 0.713 \\
\hline
\end{tabular}

*Fisher's exact test.

Table 5

Absolute quantity accuracy of pedicle screws

\begin{tabular}{|llll|}
\hline & $\begin{array}{l}\text { NM-TLIF } \\
(\mathrm{n}=112)\end{array}$ & $\begin{array}{l}\text { N-TLIF } \\
(\mathrm{n}=104)\end{array}$ & $\mathrm{p}$-Value \\
\hline Axial translational error $(\mathrm{mm})$ & $0.82 \pm 2.77$ & $-0.33 \pm 0.76$ & 0.000 \\
\hline Axial angular error (degree) & $0.49 \pm 5.62$ & $0.14 \pm 4.27$ & 0.862 \\
\hline Sagittal translational error $(\mathrm{mm})$ & $-0.89 \pm 2.04$ & $0.16 \pm 1.03$ & 0.000 \\
\hline Sagittal angular error (degree) & $1.34 \pm 4.79$ & $-0.83 \pm 3.12$ & 0.000 \\
\hline
\end{tabular}

Two-Sample Mann-Whitney U Test. 


\section{Figures}
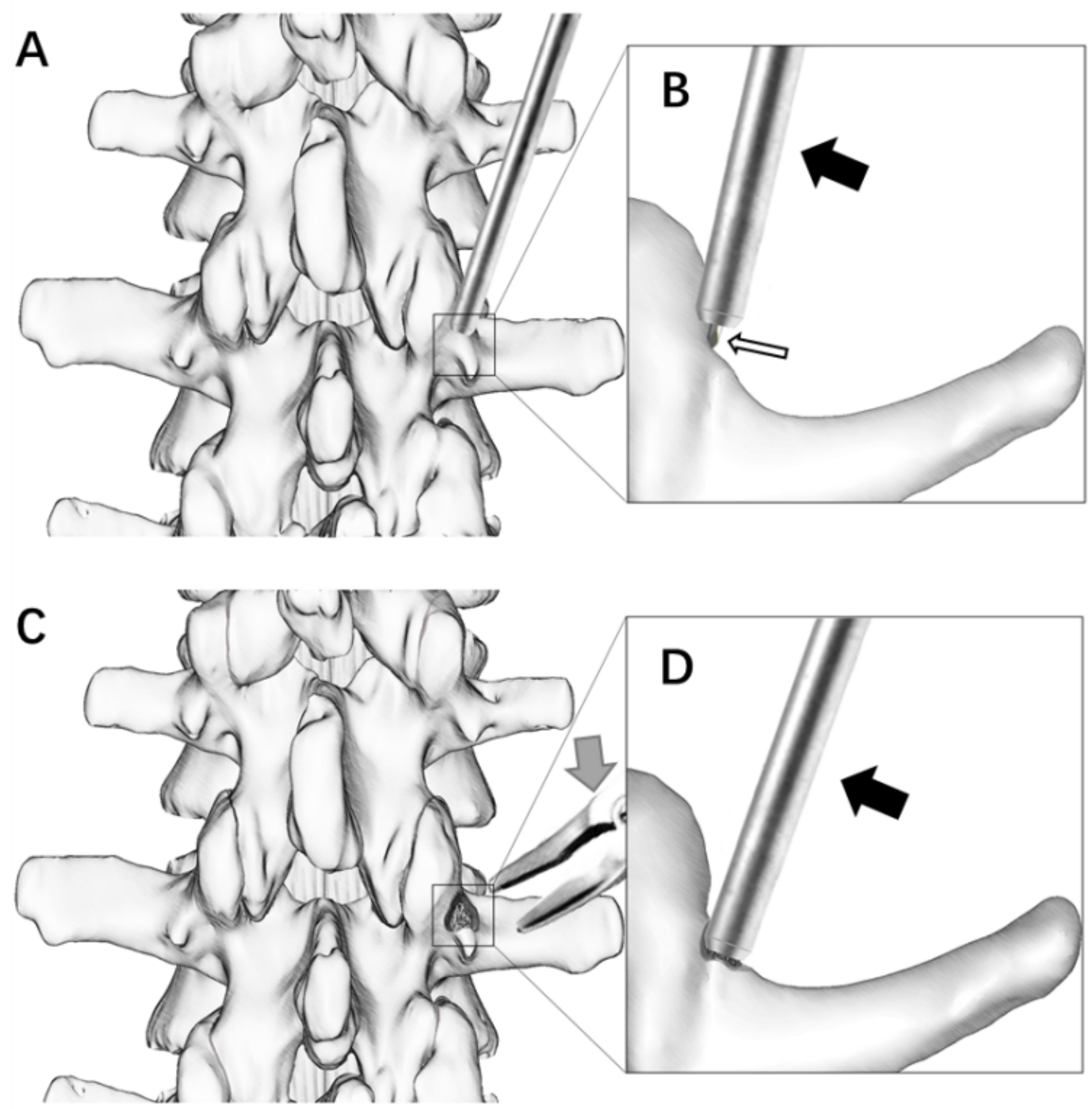

Figure 1

Difference of two methods of pedicle screw implantation. (A) Posterior view of the spine. The cortical bone could not be excised under minimally invasive surgery, and navigation drill guide (NDG) was placed in the planned ideal insertion location and is drilled with a Kirschner wire to obtain the trajectory. (B) Axial view of the spine. The NDG at the ideal insertion location is on the inclined plane of the cortical bone. (C) 
Posterior view of the spine. In open surgery, the exact insertion point can be obtained by removing a piece of cortical bone with a rongeur. (D) Axial view of the spine. After the removal of the cortical bone, the NDG can be firmly anchored to the obtained relatively flat surface. Black arrow: navigation drill guide, NDG. Grey arrow: rongeur. White arrow: kirschner wire

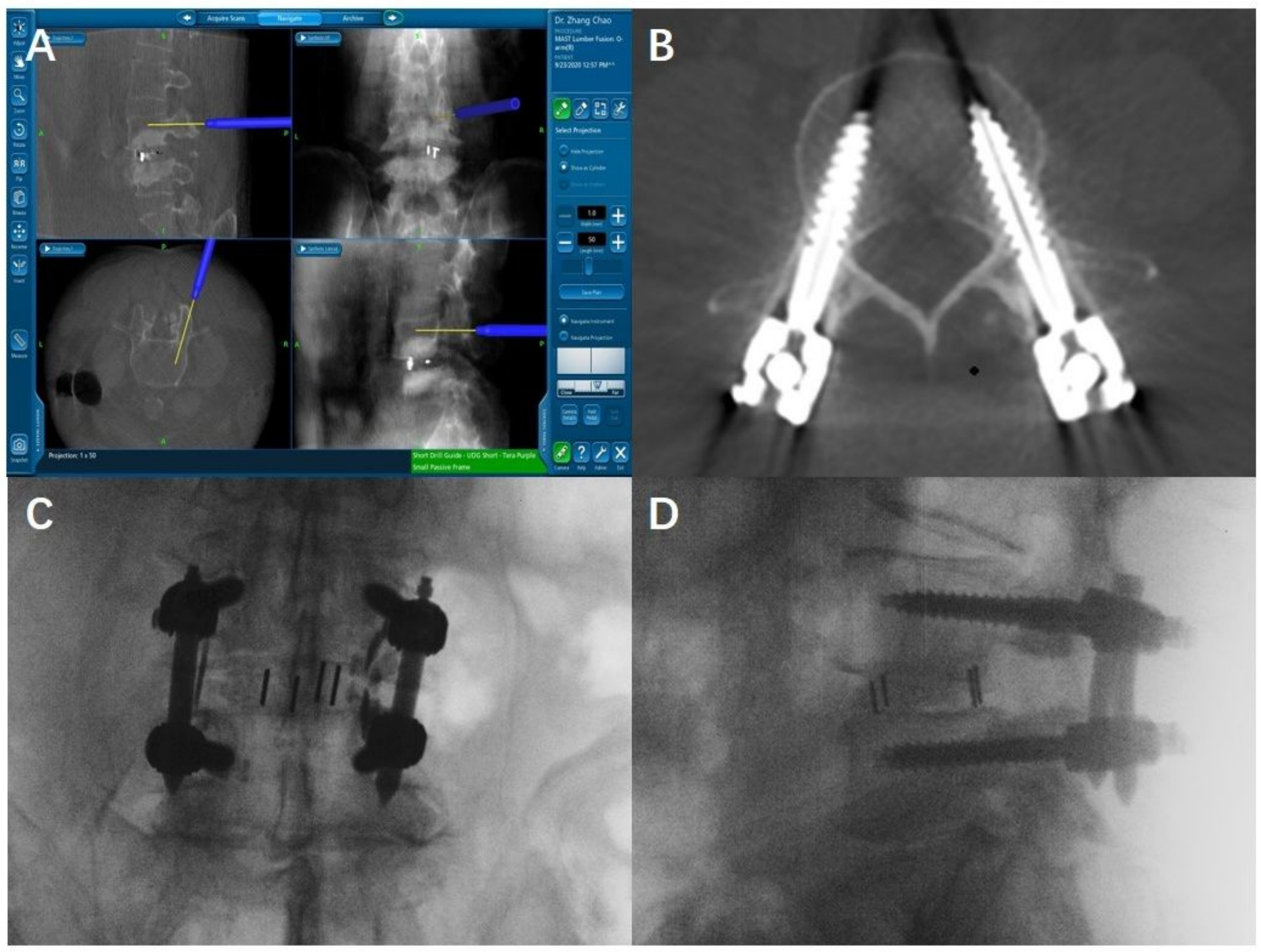

\section{Figure 2}

Intraoperative planning and outcomes. (A) A screenshot of the intraoperative planning of navigation, showing the sagittal and axial views of the planned insertion trajectory, and the positive and lateral views of the reconstructed fluoroscopy. (B) Postoperative CT reconstruction shows that the pedicle screws are completely within the vertebral pedicle(C) A typical anterior view of a navigation-guided pedicle screw implant. (D) A typical lateral view of a navigation-guided pedicle screw implant. 


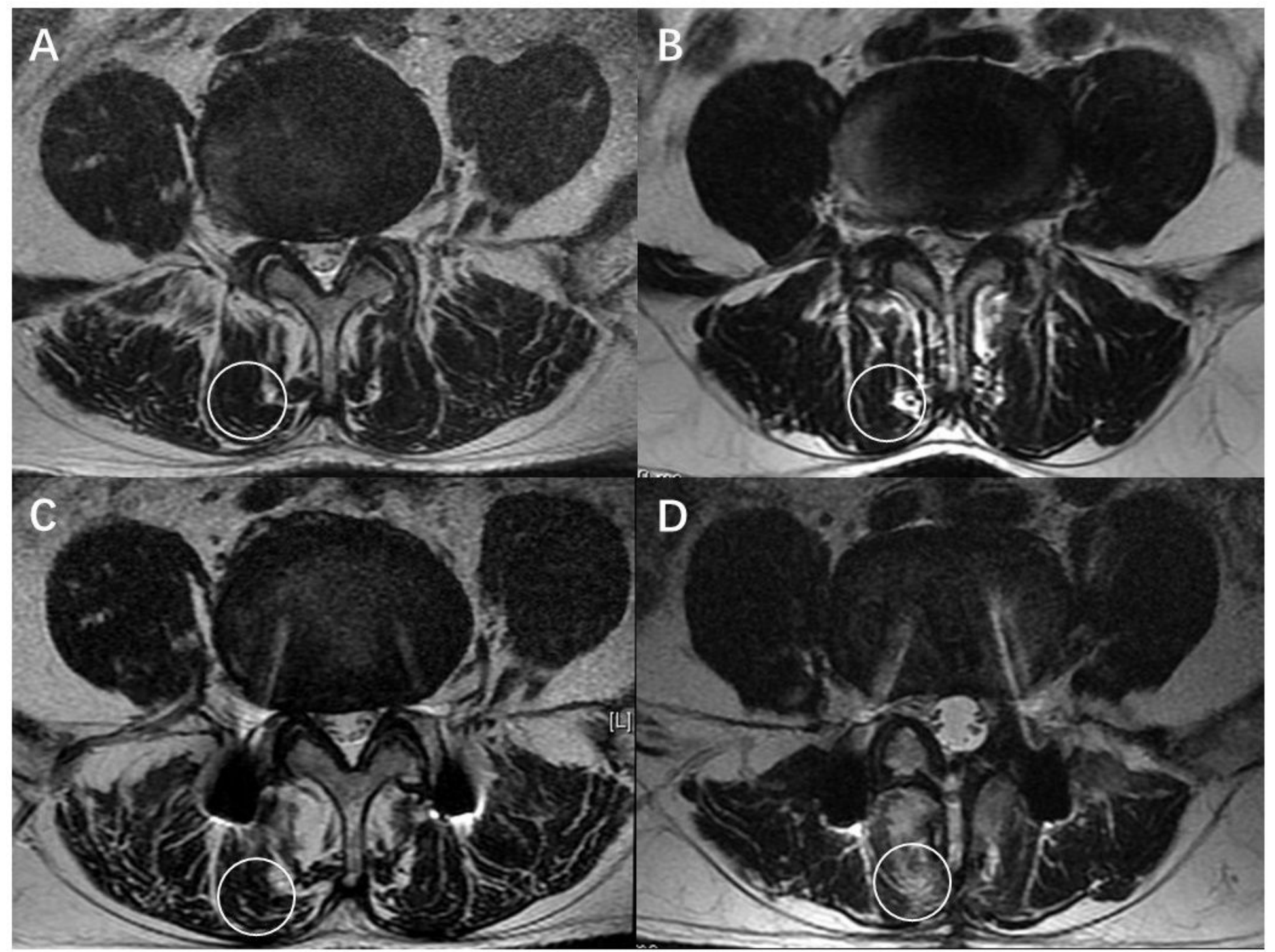

\section{Figure 3}

One-year follow-up MRI comparison. (A+C) MRI of a 57 years female preoperatively and one-year followup in NM-TLIF group. $(B+D)$ MRI of a 55 years female preoperatively and one-year follow-up in N-TLIF group. Preoperative and one-year follow-up MRI images showed that the degree of multifidus atrophy in the NM-TLIF group was significantly lower than that in the N-TLIF group. Circle『Region of interest $\mathbb{Z}$ $300 \mathrm{~mm} 2$ 


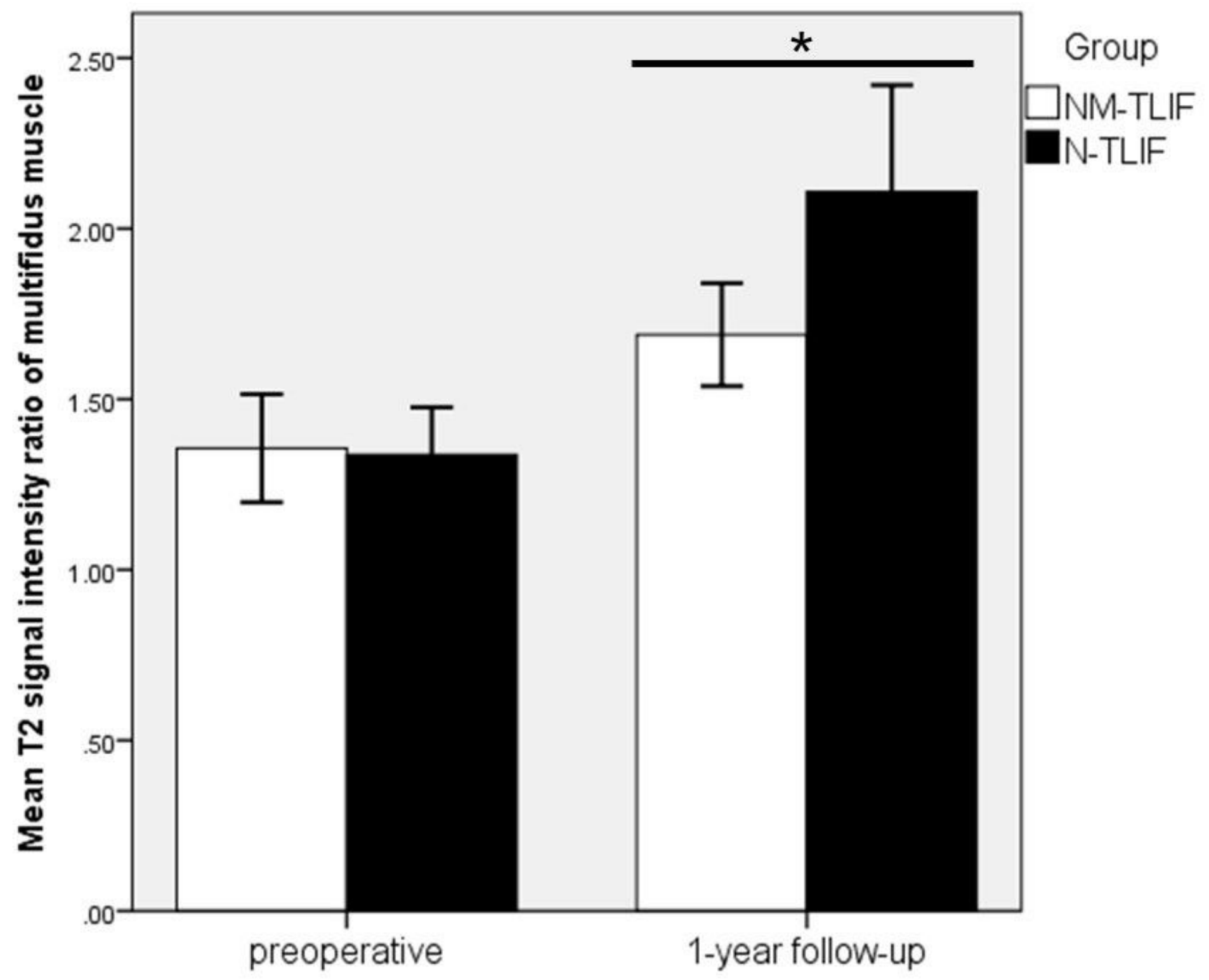

Error bars: $95 \% \mathrm{Cl}$

Figure 4

Mean MRI T2-weighted signal intensity ratio of multifidus muscle. Bar graph showing preoperative and 1year follow-up differences in mean MRI T2 signal intensity ratio of multifidus muscle between the 2 groups. The mean MRI T2 signal intensity ratio of multifidus muscle of the NM-TLIF group was significantly lower than the N-TLIF group at 1-year follow-up $(P=0.022)$ * ${ }^{*}$-value $<0.05$ 